Pacific Journal of Mathematics

RATIONAL EXTENSIONS OF MODULES 


\title{
RATIONAL EXTENSIONS OF MODULES
}

\author{
HANS H. STORRER
}

It is shown, that a module $B$ is a rational extension of a submodule $A$ if and only if $B / A$ is a torsion module with respect to the largest torsion theory for which $B$ is torsionfree. The rational completion of a module can thus be viewed as a module of quotients. The behavior of rationally complete modules under the formation of direct sums and products is studied. It is also shown, that a module is rationally complete provided it contains a copy of every nonprojective simple module.

In the second part of the paper, rational extensions of modules over a left perfect ring are studied. Necessary and sufficient conditions are given for a semi-simple module to be rationally complete. This characterization depends only on the idempotents of the ring. If $R$ is left and right perfect and if every simple right module is rationally complete, then every module is rationally complete.

1. Filters and rational extensions. We first recall a number of definitions and results concerning filters of ideals and torsion theories. Our main reference is Lambek [15], whose terminology we follow. The reader may wish to consult some related papers, e.g., $[6,11,12,16,17]$.

All rings have a unit element, all modules are unital and, unless otherwise stated, "module" means "right $R$-module".

A set $\mathfrak{F}$ of right ideals of $R$ is called a filter, if the following conditions are satisfied:

(1) Every right ideal containing a member of $\mathfrak{F}$ belongs to $\mathfrak{F}$.

(2) $\mathfrak{F}$ is closed under finite intersections.

(3) If $I \in \mathfrak{F}$ and $r \in R$, then $r^{-1} I=\{x \in R \mid r x \in I\} \in \mathfrak{F}$. The filter $\widetilde{F}$ is called idempotent, if.

(4) If $I \in \mathfrak{F}$ and $J$ is a right ideal, such that $a^{-1} J \in \mathfrak{F}$ for all $a \in I$, then $J \in \mathfrak{F}$.

The set of filters is partially ordered by inclusion. The filter $\widetilde{\mho}_{0}$ consisting of $R$ alone is the smallest, the filter $\widetilde{F}_{\infty}$ consisting of all right ideals is the largest filter. Both are idempotent.

Let $\mathfrak{F}$ be an idempotent filter and $M$ a module. We define the $\widetilde{\Im}$-torsion submodule $\mathfrak{F}(M)$ to be the set of all $m \in M$, whose annihilator is in $\mathfrak{F} . \quad M$ is said to be $\mathfrak{F}$-torsion if $\mathfrak{F}(M)=M$ and $\mathfrak{F}$-torsionfree if $\mathfrak{F}(M)=0$. The module $M / \mathfrak{F}(M)$ is always $\mathfrak{F}$-torsion-free.

A module is called $\widetilde{\Im}$-divisible if every homomorphism from $I$ to $M$, where $I \in \mathfrak{F}$ can be extended to a homomorphism from $R$ to $M$ 
or, equivalently, if $E(M) / M$ is $\widetilde{f}$-torsion-free. Here and throughout the paper, $E(M)$ denotes the injective hull of $M$.

If $M$ is $\mathfrak{F}$-torsion-free, then there is a unique (up to isomorphism) module $Q_{\mathfrak{F}}(M)$ (hereafter denoted by $Q$ ) containing $M$ such that (i)

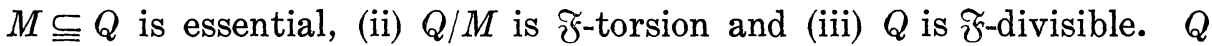
is $\mathfrak{F}$-torsion-free and it is given by the formula $Q / M=\mathfrak{F}(E(M) / M)$ or, explicitly, $Q=\left\{x \in E(M) \mid x^{-1} M \in \mathfrak{F}\right\}$, where $x^{-1} M=\{r \mid x r \in M\}$. For an arbitrary $M$, we define $Q_{\Re}(M)$ to be $Q_{\mathfrak{F}}(M / \widetilde{F}(M))$. It can be shown, that $Q_{\mathfrak{F}}(M)=\lim _{\rightarrow} \operatorname{Hom}_{R}(I, M / \mathfrak{F}(M))(I \in \mathfrak{\xi}) . \quad Q$ is called the module of quotients of $M$ with respect to $\widetilde{r}$.

Given any $M$, the set $\widetilde{\mho}_{M}=\left\{I \mid \operatorname{Hom}_{R}(R / I, E(M))=0\right\}$ is an idempotent filter and it is the largest among all idempotent filters (S) such that $M$ is (S)-torsion-free.

Proposition 1.1.

(a) $I \in \mathfrak{F}_{M}$ if and only if $x I \neq 0$ for every $x \in E(M), x \neq 0$.

(b) $I \in \widetilde{F}_{M}$ if and only if for all $m \in M, m \neq 0$, and all $r \in R$ there is an $s \in R$ such that $m s \neq 0, r s \in I$.

Proof. (a) There is a nonzero map $R / I \rightarrow E(M)$ if and only if there is an $x \in E(M), x \neq 0$, such that $x I=0$. (b) is proved in [15, Prop. 0.2].

A module $P$ is $\widetilde{F}_{M}$-torsion if and only if $\operatorname{Hom}_{R}(P, E(M))=0$. As an example, consider $\mathfrak{F}_{R}=\mathfrak{F}_{E(R)}$. This is the filter of dense right ideals $[14$, p. 96$]$.

LEMMA 1.2.

(a) $L \subseteq M$ implies $\mathfrak{F}_{L} \supseteqq \widetilde{\mho}_{M}$.

(b) If $L \subseteq M$ is an essential extension, then $\widetilde{\mho}_{L}=\widetilde{\mho}_{M}$.

(c) If $\left\{M_{\alpha}\right\}$ is an arbitrary family of modules and if $S=\sum_{\alpha} M_{\alpha}$, $P=\Pi_{\alpha} M_{\alpha}$, then $\mathfrak{F}_{S}=\mathfrak{F}_{P}=\bigcap_{\alpha} \mathfrak{F}_{M_{\alpha}}$.

The proof is straightforward by $(1.1, b)$.

In [9], Findlay and Lambek define a relation between three modules. The write $A \leqq B(M)$ if $A \subseteq B$ and if for every homomorphism $\phi: C \rightarrow M$, where $A \leqq C \leqq B, \phi(A)=0$ implies $\phi=0$. Equivalently, $A \leqq B(M)$ if and only if $\operatorname{Hom}_{R}(B / A, E(M))=0$, i.e., if $B / A$ is $\widetilde{\mho}_{M}$-torsion.

$A \subseteq B$ is called a rational extension if $A \leqq B(B)$. In view of the remarks above and [9, Prop. 2.2], we have.

LEMMA 1.3. The following statements are equivalent:

(a) $A \subseteq B$ is a rational extension.

(b) $A \subseteq B$ is an essential extension and $B / A$ is $\mathfrak{F}_{A}$-torsion.

(c) $A \subseteq B$ and $B / A$ is $\widetilde{\mho}_{B}$-torsion. 
A module is called rationally complete if it has no proper rational extensions. In particular, an injective module is rationally complete. Findlay and Lambek [9] have shown, that every module has a maximal rational extension $\bar{M}$ which is rationally complete. $\bar{M}$ is unique up to isomorphism and it is called the rational completion of $M$.

Proposition 1.4.

(a) $\bar{M}$ is the module of quotients $Q_{\mathfrak{\Im}_{M}}(M)$,

(b) $\bar{M}=\left\{x \in E(M) \mid x^{-1} M \in \mathfrak{F}_{M}\right\}$.

Proof. By (1.3) $\bar{M}$ satisfies properties (i) and (ii) of the module of quotients. Also by (1.3) $\bar{M} \subseteq E(M)$. Let now $T / \bar{M}$ be the $\widetilde{F}_{M^{-}}$ torsion-submodule of $E(M) / \bar{M}$, then $T$ is a rational extension of $\bar{M}$ by (1.3), since $\widetilde{\mho}_{M}=\widetilde{\mho}_{T}$. But $\bar{M}$ has no proper rational extensions, hence $T=\bar{M}$ and $E(M) / \bar{M}$ is $\widetilde{\mho}_{M}$-torsion-free, thus satisfying condition (iii). (b) is just the explicit description.

CoRollary 1.5. A module $M$ is rationally complete if and only if $M$ is $\widetilde{\mho}_{M}$-divisible.

Proposition 1.6. (Brown [4]). The direct product of rationally complete modules is rationally complete.

Proof. Let $P=\Pi_{\alpha} M_{\alpha}$ and suppose each $M_{\alpha}$ is rationally complete. By $\left(1.2\right.$, c) $\widetilde{\mho}_{P} \subseteq \widetilde{\mho}_{M_{\alpha}}$ for each $\alpha$. Since each $M_{\alpha}$ is $\widetilde{\mho}_{P}$-divisible, $P$ is also $\widetilde{\mho}_{P}$-divisible.

Following Goldman, [12], a filter $\mathfrak{F}$ is called Noetherian, if it has the following property: If $I_{1} \subseteq I_{2} \subseteq \cdots$ is a (countable) ascending chain of right ideals whose union is in $\mathfrak{F}$, then some $I_{n}$ is in $\mathfrak{F}$. This condition is satisfied, if every right ideal in $\widetilde{f}$ contains a finitely generated right ideal also in $\widetilde{\wp}$.

Proposition 1.7. The direct sum of any family of rationally complete modules is rationally complete if and only if every idempotent filter is Noetherian.

Proof. Let $\left\{M_{\alpha}\right\}$ be a family of rationally complete modules and $S$ be their sum. Then $\mathfrak{F}_{S}=\cap_{\alpha} \widetilde{\mho}_{M_{\alpha}}$ by (1.2) and each $M_{\alpha}$ is $\widetilde{F}_{s}$-torsionfree and $\mathfrak{F}_{s}$-divisible. Since $\mathfrak{F}_{s}$ is Noetherian by assumption, $S$ is $\widetilde{F}_{s}$-divisible by [12, Thm. 4.4], hence rationally complete by (1.5).

Conversely, suppose there is a filter $\widetilde{F}$ which is not Noetherian. Then there is a right ideal $I \in \widetilde{F}$ such that $I$ is the union of a chain $I_{1} \subseteq I_{2} \subseteq \cdots$, where no $I_{i}$ is in $\widetilde{F}$.

Let $M$ be an injective module such that $\mathfrak{\mho}=\mathfrak{F}_{M}$. Such a module 
exists by [12, Thm. 5.3]. $M$ is rationally complete, but we claim, that the sum $S$ of countably many copies of $M$ is not rationally complete. By assumption. $I_{i} \notin \widetilde{\mho}_{M}$ for all $\mathrm{i}$, hence by $(1.1, \mathrm{a})$, there exist nonzero elements $x_{i} \in M$ such that $x_{i} I_{i}=0$. If $d \in I$, then $d \in I_{k}$ for some $k$, hence $x_{i} d=0$ for $i \geqq k$ and $\phi(d)=\left(\cdots, x_{i} d, \cdots\right)$ defines a homomorphism $I \rightarrow S$. Suppose that $S$ is rationally complete, then $\phi$ extends to a map $\phi^{\prime}: R \rightarrow S$ and $\phi^{\prime}(1)=\left(y_{1}, y_{2}, \cdots, y_{n}, 0,0, \cdots\right)$. Thus $x_{i} I=0$ for all $i>n$, contrary to the assumption.

The proof shows a little bit more: If every injective (or rationally complete) module $M$ is countably $\Sigma$-rationally complete (i.e., a countable sum of copies of $M$ is rationally complete), then the sum of any family of rationally complete modules is rationally complete. This is an analogue of the situation for injective modules [8, p. 205].

It is not true in general that a direct summand of a rationally complete module is rationally complete (see (1.9) below). However, we have

Proposition 1.8. Let $\left\{M_{\alpha}\right\}$ be a family of modules such that

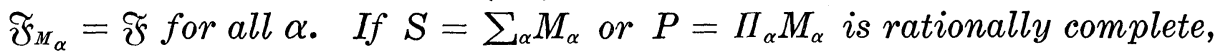
then $M_{\alpha}$ is rationally complete for every $\alpha$.

Proof. Let $\phi: I \rightarrow M_{\alpha}$ be a homomorphism; where $I \in \widetilde{\mho}_{M_{\alpha} \cdot}$ Because $\widetilde{\mho}_{M_{\alpha}}=\widetilde{F}_{P}$ by $(1.2) \phi$ extends to $\phi^{\prime}: R \rightarrow P$ (after some identifications) and $\pi_{\alpha} \phi^{\prime}: R \rightarrow M_{\alpha}$ is the desired extension of $\phi$, where $\pi_{\alpha}$ is the projection $P \rightarrow M_{\alpha}$. The proof for $S$ is similar.

Proposition 1.9. Let $\left\{S_{\alpha}\right\}$ be a set of nonisomorphic simple modules, representing all nonprojective simple modules. Then every module containing the module $T=\sum_{\alpha} S_{\alpha}$ is rationally complete.

Proof. First note that a simple module $S \cong R / A$ ( $A$ a maximal right ideal) is projective if and only of it is isomorphic to a direct summand of $R$ or if and only if $A$ is a direct summand of $R$.

Suppose now $T \subseteq M$. Suppose $x \in \bar{M} \subseteq E(M)$, then $I=x^{-1} M \in \mathfrak{F}_{M}$. Since $E(M)$ is an essential extention of $M, I$ is a large [14, p. 70] right ideal of $R$. Suppose $I \neq R$, then $I$ is contained in a maximal right ideal $A$, which is also large. Furthermore, $A \in \mathfrak{F}_{M}$. The simple module $S=R / A$ is not projective by what has been said above, hence there is a nonzero homomorphism $R / A \rightarrow T \rightarrow M \rightarrow E(M)$, which contradicts the fact that $A \in \widetilde{\mho}_{M}$. Hence $I=R$ and $x \in M$, i.e., $M=\bar{M}$.

A similar result was proved in [4] with $T=\sum R / L, L$ running through all large right ideals.

An immediate consequence is that the class of rationally complete modules is closed under submodules (or factor modules) if and only 
if every module is rationally complete. A characterization of the rings with the latter property is given in [5]. As Brown [4] has remarked, (1.9) also implies that every rationally complete module is injective if and only if $R$ is completely reducible (i.e., semisimple Artinian). We also have

CoRollaRY 1.10. Every rationally complete module is projective if and only if $R$ is completely reducible.

Proof. Choose one simple module from each isomorphism class and let $U$ be their sum. Then $U$ is rationally complete by (1.9), hence every simple module is projective. Therefore $R$ has no proper large ideal and $R$ is completely reducible by $[14, \mathrm{p} .61]$. The converse is obvious.

CoROLLARY 1.11. Every cogenerator is rationally complete.

Proof. A cogenerator contains a copy of every simple module.

Proposition 1.12. Suppose every simple module is isomorphic to a minimal right ideal. Then every faithful module is rationally complete.

Proof. If $M$ is faithful, then $R \subseteq \Pi M$ for some index set. Since $T \subseteq R$ by assumption, $\Pi M$ is rationally complete and hence so is $M$ by (1.8).

One might ask whether any module $S$ with the property that $S \oplus M$ is rationally complete for all $M$, has to contain the module $T$ defined above. This is not so in general but the following discussion shows that this situation arises for the ring $\mathbf{Z}$ of integers.

EXAMPLE 1.13. Let us first describe the idempotent filters of Z. Given any subset $\mathscr{P}$ of the set of all primes, the ideals generated by products of powers of primes from $\mathscr{P}$ form an idempotent filter. Conversely, any idempotent filter (except $\mathfrak{F}_{\infty}$ ) is of this form. This follows from unique factorization and the fact that in a commutative Noetherian ring a filter $\mathfrak{F}$ is idempotent if and only if $I, J \in \mathfrak{F}$ implies $I J \in \widetilde{\mho}[17,1.22]$.

If $M$ is any nonzero Abelian group, we let $\mathscr{P}$ be the set of primes, such that $p m=0(p \in \mathscr{P}, m \in M)$ implies $m=0$. (Thus $M$ is $\mathscr{P}$-torsion-free in the sense of [13]). $\widetilde{\mho}_{M}$ is then the filter "generated" by $\mathscr{P}$. By (1.5) $M$ is rationally complete if and only if $M$ is divisible by all primes in $\mathscr{P}$ (.P -divisible [13]). In particular, any torsion group is rationally complete and a torsion-free group is rationally 
complete if and only if it is divisible.

The group $T$ is the sum of all $\mathbf{Z} /(p)$. We claim that $T$ is the smallest group such that $T \oplus M$ is rationally complete for all $M$. Indeed, suppose $U$ does not contain $Z /(q)$. Then $U$ is $q$-torsion-free and so is $U \oplus \mathbf{Z}$. But $U \oplus \mathbf{Z}$ is not $q$-divisible, hence not rationally complete.

2. Rational extensions of modules orer left perfect rings. From now on $R$ shall denote a left perfect ring [3] with Jacobson radical $N$. We shall use the following properties of $R$ : Every right $R$-module $M$ has nonzero socle Soc $M$ and $R$ satisfies the minimum condition on principal right ideals. Furthermore, the following facts, which are well-known for Artinian rings [2] are also true for perfect rings: The unit element of $R$ can be written as a sum of orthogonal primitive idempotents. If $e$ is any primitive idempotent, then $e R$ has a unique maximal submodule $e N$, hence $e R / e N$ is simple. Every simple module $S$ is of this form: $S \cong e R / e N$ if and only if $S e \neq 0$. Two primitive idempotents $e$ and $f$ are called isomorphic if $e R \cong f R$, or equivalently if $e R / e N \cong f R / f N$. Furthermore $e$ and $f$ are isomorphic if and only if there exist $u, v \in R$ such that $e=u f v$ and $f=v e u$ $[14$, p. 63], this shows that the concept is left-right symmetric.

From now on, we consider a fixed representation of the unit element as a sum of orthogonal primitive idempotents.

$$
1=e_{11}+\cdots+e_{1 k_{1}}+\cdots+e_{n 1}+\cdots+e_{n k_{n}},
$$

where $e_{i j}$ is isomorphic to $e_{r s}$ if and only if $i=r$. We also set $e_{i}=e_{i 1}$.

Once $(*)$ is fixed there is, for any simple module $S$, a unique primitive idempotent $e_{i}$ such that $S e_{i} \neq 0$. We shall say, that $e_{i}$ corresponds to $S$. If $M$ is any module, we let $e$ be the sum of the $e_{i}$ corresponding to the simple submodules of $M$. Again we say, that $e$ corresponds to $M$.

Lemma 2.1. Let $e$ correspond to $M$ and let $m \in M$. Then $m R e=0$ implies $m=0$.

Proof. If $m \neq 0$, then there is an $r \in R$ such that $0 \neq m r \in$ Soc $M$. Thus $x r R$ is a sum of simple submodules of $M$ and $x r R e \neq 0$.

LEMMA 2.2. Let $S$ be a simple module with corresponding idempotent $e_{i}$ and let $J$ be a two-sided ideal. Then $S J=S$ if and only if $e_{i} \in J$.

Proof. The "if" part is clear. To prove the "only if" part suppose $S J \neq 0$. Now $J=f R+P$ where $f^{2}=f$ and $P \subseteq N$. (See e.g., 
[2, Thm. $2.6 \mathrm{~B}$ ] for a proof in the Artinian case). $f$ can be written as a sum of primitive idempotents $f_{j} \in J$ and since $S P=0$, it follows that $S f_{k} \neq 0$ for some $k$. Therefore $f_{k}$ is isomorphic to $e_{i}$ and since $J$ is two-sided $e_{i} \in J$.

Proposition 2.3. Let $M$ be any module over the left perfect ring $R$ and let $e$ correspond to $M$. Then $\mathfrak{F}_{M}$ consists of all right ideals containing ReR.

Proof. Suppose $R e R \subseteq I$. Since $e$ corresponds also to $E(M)$, (2.1) implies that $x I \neq 0$ for any nonzero $x \in E(M)$. Thus $I \in \mathfrak{F}_{M}$ by (1.1).

Conversely, let $I \in \mathfrak{F}_{M}$. We claim, that the two-sided ideal $J=\{r \mid R r \subseteq I\}$ is also in $\widetilde{\mho}_{M}$. To prove this, it will be sufficient to show, that $K=\{r \mid x J=0 \Rightarrow x r=0$ for all $x \in E(M)\}$ equals $R$. Suppose $K \neq R$, then we can choose an $a \notin K$, such that $a R$ is minimal in the set $\{c R \mid c \notin K\}$ of principal right ideals. Then $a \notin J$, hence there exists a $b$ such that $b a \notin I$. If $a s \notin K$ for some $s \in S$, then $a s R=$ $a R$ by minimality. Thus bas $\in I$ implies $a s \in K$ (for if not, $b a \in I$ by the preceding remark) and we conclude that $(b a)^{-1} I \subseteq a^{-1} K=L$ and $L \in \widetilde{\mho}_{M}$. Since $a L \subseteq K, x J=0$ implies $x a L=0$ and hence $x a=0$ by $(1.1, a)$, contradicting the assumption that $a \notin K$.

Since $J \in \widetilde{F}_{M}, S J \neq 0$ for every simple submodule $S$ of $M$. Lemma (2.2) implies then $e \in J$ and it follows that $R e R \subseteq J \subseteq I$.

COROLLARY 2.4. Every idempotent filter of the left perfect ring $R$ is of the form $\{I \mid R e R \subseteq I\}$ for some idempotent $e$.

Proof. By [12, Thm. 5.3], every idempotent filter is of the form $\widetilde{\mho}_{M} \cdot$

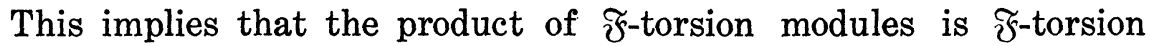
for any $\mathfrak{~}$. See [1], [7].

We also point out, that $R e R$, where $e=e_{i_{1}}+\cdots+e_{i_{k}}$ depends only on the isomorphism classes of the $e_{i_{j}}$. Furthermore $R e R$ contains every idempotent isomorphic to one of the $e_{i}$. Thus, the left perfect ring $R$ possesses $2^{n}$ idempotent filters of left ideals, where $n$ is the number of isomorphism classes of simple modules.

We shall use (2.3) to describe the rational completion $\bar{M}$ of a module $M$ over a left perfect ring $R$. Note that if $\mathfrak{F}=\{I \mid \operatorname{Re} R \subseteq I\}$, then the $\mathfrak{F}$-torsion-submodule $\mathfrak{F}(M)$ of $M$ is given by

$$
\mathfrak{F}(M)=\{m \mid m R e=0\} \text {. }
$$

Proposition 2.5. Let $R$ be left perfect and let $M$ be a module 
with corresponding idempotent $e$.

(a) $\bar{M}=\{x \in E(M) \mid x R e \subseteq M\}$

(b) If $M$ is semi-simple, then $\bar{M}=\{x \in E(M) \mid x R e N=0\}$.

Proof. (a) follows from $(1.4, b)$ and $(2.3)$. To prove (b), note that $x R e \subseteq M$ if and only if $x R e N=0$.

Lemma 2.6. Let $M$ be any module with corresponding idempotent e. Let $I, J$ be right ideals of $R$. Then

(a) $\{x \in E(M) \mid x I=0\}=\{x \in E(M) \mid x I e=0\}$

(b) Suppose that $I \subseteq J$ and that

$$
\{x \in E(M) \mid x I=0\}=\{x \in E(M) \mid x J=0\} .
$$

Then $I e=J e$.

Proof. (a) This is the first part of Lemma 1.1, (a) in [10]. The proof given there still works in the present, slightly more general case.

(b) Suppose $I e \neq J e$. Then $(J / I) e \neq 0$, hence there is a $y \in J / I$ such that $y e_{i} \neq 0$ for some primitive idempotent $e_{i}$ corresponding to a simple submodule of $M$. The module $y e_{i} R$ is a nonzero homomorphic image of $e_{i} R$ and therefore maps onto $e_{i} R / e_{i} N$. It follows, that there are right ideals $A, B$ with $I \subseteq B \cong A \subseteq J$ such that

$$
A / B \cong e_{i} R / e_{i} N \cong E(M) \text {. }
$$

Thus, there is a nonzero homomorphism $A \rightarrow A / B \rightarrow E(M)$, which extends to a nonzero $\phi: R \rightarrow E(M)$ given by $\phi(1)=x \neq 0$. Now $B \subseteq$ Ker $\phi$, hence $x I=0$, but $\phi(A) \neq 0$, hence $x J \neq 0$. This contradicts the assumption.

Proposition 2.7. Let $R$ be left perfect and let $M$ be a semisimple module with corresponding idempotent $e$. Then

(a) $\bar{M}=E(M)$ if and only if $e N e=0$,

(b) $\bar{M}=M$ (i.e., $M$ is rationally complete) if and only if $R e N e=N e$,

(c) $M=E(M)$ (i.e., $M$ is injective) if and only if $\mathrm{Ne}=0$,

(d) $M$ is projective if and only if $e N=0$.

Proof. (a) If $\bar{M}=E(M)$ we have by (2.5)

$$
E(M)=\{x \in E(M) \mid x \cdot 0=0\}=\bar{M}=\{x \in E(M), x R e N=0\} .
$$

Applying $(2.6, b)$ to $\mathrm{I}=0, J=R e N$, we get that $R e N e=0$, which is equivalent to $e N e=0$. Conversely, if $R e N e=0$, then $(2.6$, a) applied 
to $I=R e N$, yields $\bar{M}=E(M)$.

(b) Suppose $\bar{M}=M$. Since $M$ is the socle of $E(M)$,

$$
M=\{x \in E(M) \mid x N=0\} .
$$

Applying $(2.6, \mathrm{~b})$ again, this time to $I=R e N, J=N$, we obtain $R e N e=N e$. The converse is proved as above.

(c) This follows from (a) and (b).

(d) A simple module $e_{i} R / e_{i} N$ is projective if and only if $e_{i} N=0$.

COROLLARY 2.8. If the rational completion of every semisimple module is injective, then $R$ is completely reducible.

Proof. Let $M$ be a semi-simple module with corresponding idempotent $e=e_{1}+\cdots+e_{n}$. Then $R e R=R$ and $e N e=0$ implies $N=0$.

The ring of $2 \times 2$ triangular matrices over a field is not completely reducible, yet the rational completion of every simple module is injective.

Another immediate consequence of (2.7) is the following.

CoRollary 2.9. A projective semi-simple module is rationally complete if and only if it is injective.

A ring is called primary if it is a simple Artinian ring modulo its radical.

Proposition 2.10. Let $R$ be left and right perfect. Then the following are equivalent:

(a) Every right $R$-module is rationally complete.

(b) Every simple right $R$-module is rationally complete.

(c) $R$ is a finite product of primary left and right perfect rings.

Proof. $\quad(a) \Rightarrow(b)$ is trivial. (c) $\Rightarrow$ (a) is a part of [5, Main Theorem]. It remains to prove $(b) \Rightarrow(c)$.

Using the decomposition $\left({ }^{*}\right)$, we set $f_{i}=e_{i 1}+\cdots+e_{i k_{i}}$ and, as before, $e_{i}=e_{i 1}$ for $i=1, \cdots, n$. By assumption and (2.7), we have that $R e_{i} N e_{i}=N e_{i}$ for all $i$. This implies $R f_{i} N f_{i}=N f_{i}$ for all $i$, because $e_{i}=u e_{i j} v$ and $e_{i j}=v e_{i} u$ for some $u, v \in R$, whence $R e_{i} N=R f_{i} N$.

Let now $e$ and $f$ be any two different idempotents from the set $\left\{f_{i}\right\}$. Since $e R f$ annihilates every simple $R$-module, we have that $e R f \subseteq N$. From this and $R f N f=N f$ it follows that

$$
R e R f R=(\operatorname{Re} R f R) N f \text {. }
$$

However, since $R$ is right perfect, a generalized version of Nakayama's 
Lemma holds: $\quad M N=M$ implies $M=0$ for every right module $M$ (see [3, p.473]). Thus $e R f=0$ and we conclude that all idempotents $f_{i}$ are central. This implies (c).

\section{REMARKS 2.11.}

(a) Since $(2.10, \mathrm{c})$ is left-right symmetric, Proposition (2.10) is also true for left modules instead of right modules.

(b) There exist rings such that every simple module but not every module is rationally complete; e.g., the ring $\boldsymbol{Z}$ of integers (see (1.13)). As a matter of fact, Brown [4] has shown that every simple module over a commutative ring is rationally complete.

\section{REFERENCES}

1. J. S. Alin and E. P. Armendariz, TTF-classes over perfect rings, J. Austral. Math. Soc., (to appear).

2. E. Artin, C. J. Nesbitt and R. M. Thrall, Rings with Minimum Condition, Ann Arbor: The University of Michigan Press, 1943.

3. H. Bass, Finitistic dimension and homological generalization of semi-primary rings, Trans. Amer. Math. Soc., 95 (1960), 466-488.

4. S. H. Brown, On Rationally Complete Modules, Ph.D. Thesis, North Carolina State University, Raleigh, N. C., 1969.

5. R. Courter, Finite direct sums of complete matrix rings over perfect completely primary rings, Canad. J. Math., 21 (1969), 430-446.

6. S. E. Dickson, A torsion theory for Abelian categories, Trans. Amer. Math. Soc., 121 (1966), 223-235.

7. V. Dlab, A characterization of perfect rings, Pacific J. Math., 33, (1970), 79-88.

8. C. Faith, and E. A. Walker, Direct-sum representation of injective modules, J. Algebra, 5 (1967), 203-221.

9. G. D. Findlay, and J. Lambek, A generalized ring of quotients $I$, II, Canad. Math. Bull., 1 (1958), 77-85, 155-167.

10. K. Fuller, On indecomposable injectives over Artinian rings, Pacific J. Math., 29 (1969), 115-135.

11. P. Gabriel, Des catégories abéliennes, Bull. Soc. Math. France, 90 (1962), 323-448.

12. O. Goldman, Rings and modules of quotients, J. Algebra, 13 (1969), 10-47.

13. P. J. Hilton and S. M. Yahya, Unique divisibility in Abelian groups, Acta Math. Acad. Sci. Hung., 14 (1963), 229-239.

14. J. Lambek, Lectures on Rings and Modules, Waltham: Blaisdell 1966.

15. - Torsion Theories, Additive Semantics and Rings of Quotients, Lecture Notes in Mathematics. Berlin, Heidelberg, New York: Springer (to appear).

16. J. M. Maranda, Injective structures, Trans. Amer. Math. Soc., 110 (1964) 98-135.

17. C. L. Walker, and E. A. Walker, Quotient categories and rings of quotients, (to appear).

Received December 11, 1970. This work was supported by "Schweizerischer National fonds zur Forderung der wissenschaftlichen Forschung".

TUlane UNIVERSITY 


\title{
PACIFIC JOURNAL OF MATHEMATICS
}

\author{
EDITORS
}

\author{
H. Samelson \\ Stanford University \\ Stanford, California 94305 \\ C. R. HOBBY \\ University of Washington \\ Seattle, Washington 98105
}

J. DugundJI

Department of Mathematics

University of Southern California

Los Angeles, California 90007

RICHARD ARENS

University of California

Los Angeles, California 90024

\section{ASSOCIATE EDITORS}

E. F. BECKENBACH
B. H. NEUMANN

F. WoLF
K. YosHIDA

\section{SUPPORTING INSTITUTIONS}

\author{
UNIVERSITY OF BRITISH COLUMBIA \\ CALIFORNIA INSTITUTE OF TECHNOLOGY \\ UNIVERSITY OF CALIFORNIA \\ MONTANA STATE UNIVERSITY \\ UNIVERSITY OF NEVADA \\ NEW MEXICO STATE UNIVERSITY \\ OREGON STATE UNIVERSITY \\ UNIVERSITY OF OREGON \\ OSAKA UNIVERSITY \\ UNIVERSITY OF SOUTHERN CALIFORNIA
}

\author{
STANFORD UNIVERSITY \\ UNIVERSITY OF TOKYO \\ UNIVERSITY OF UTAH \\ WASHINGTON STATE UNIVERSITY \\ UNIVERSITY OF WASHINGTON \\ $\stackrel{*}{*} \stackrel{*}{*} \stackrel{*}{*}{ }^{*}{ }^{*}$ MERICAN MATHEMATICAL SOCIETY \\ CHEVRON RESEARCH CORPORATION \\ NAVAL WEAPONS CENTER
}

The Supporting Institutions listed above contribute to the cost of publication of this Journal, but they are not owners or publishers and have no responsibility for its content or policies.

Mathematical papers intended for publication in the Pacific Journal of Mathematics should be in typed form or offset-reproduced, (not dittoed), double spaced with large margins. Underline Greek letters in red, German in green, and script in blue. The first paragraph or two must be capable of being used separately as a synopsis of the entire paper. The editorial "we" must not be used in the synopsis, and items of the bibliography should not be cited there unless absolutely necessary, in which case they must be identified by author and Journal, rather than by item number. Manuscripts, in duplicate if possible, may be sent to any one of the four editors. Please classify according to the scheme of Math. Rev. Index to Vol. 39. All other communications to the editors should be addressed to the managing editor, Richard Arens, University of California, Los Angeles, California, 90024.

50 reprints are provided free for each article; additional copies may be obtained at cost in multiples of 50 .

The Pacific Journal of Mathematics is published monthly. Effective with Volume 16 the price per volume (3 numbers) is $\$ 8.00$; single issues, $\$ 3.00$. Special price for current issues to individual faculty members of supporting institutions and to individual members of the American Mathematical Society: $\$ 4.00$ per volume; single issues $\$ 1.50$. Back numbers are available.

Subscriptions, orders for back numbers, and changes of address should be sent to Pacific Journal of Mathematics, 103 Highland Boulevard, Berkeley, California, 94708.

PUBLISHED BY PACIFIC JOURNAL OF MATHEMATICS, A NON-PROFIT CORPORATION

Printed at Kokusai Bunken Insatsusha (International Academic Printing Co., Ltd.), 7-17, Fujimi 2-chome, Chiyoda-ku, Tokyo, Japan. 


\section{Pacific Journal of Mathematics}

J. T. Borrego, Haskell Cohen and Esmond Ernest Devun, Uniquely

representable semigroups on the two-cell .................. 565

Glen Eugene Bredon, Some examples for the fixed point property ........ 571

William Lee Bynum, Characterizations of uniform convexity .......... 577

Douglas Derry, The convex hulls of the vertices of a polygon of order $n \ldots 583$

Edwin Duda and Jack Warren Smith, Reflexive open mappings .......... 597

Y. K. Feng and M. V. Subba Rao, On the density of $(k, r)$ integers ........ 613

Irving Leonard Glicksberg and Ingemar Wik, Multipliers of quotients of

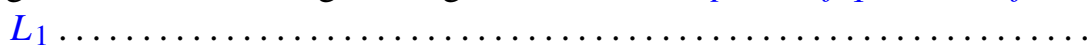

John William Green, Separating certain plane-like spaces by Peano

continua.........................................

Lawrence Albert Harris, A continuous form of Schwarz's lemma in normed

linear spaces .................................... 635

Richard Earl Hodel, Moore spaces and $w$-spaces ............... 641

Lawrence Stanislaus Husch, Jr., Homotopy groups of PL-embedding spaces.

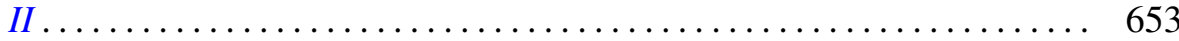

Yoshinori Isomichi, New concepts in the theory of topological

space-supercondensed set, subcondensed set, and condensed set.....

J. E. Kerlin, On algebra actions on a group algebra .................

669

Keizō Kikuchi, Canonical domains and their geometry in $C^{n} \ldots \ldots \ldots \ldots 681$

Ralph David McWilliams, On iterated $w^{*}$-sequential closure of cones. .

697

C. Robert Miers, Lie homomorphisms of operator algebras ..

717

Louise Elizabeth Moser, Elementary surgery along a torus knot ...

737

Hiroshi Onose, Oscillatory properties of solutions of even order differential

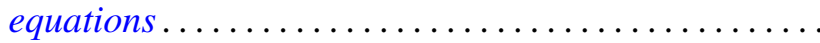

747

Wellington Ham Ow, Wiener's compactification and $\Phi$-bounded harmonic

functions in the classification of harmonic spaces...

Zalman Rubinstein, On the multivalence of a class of meromorphic

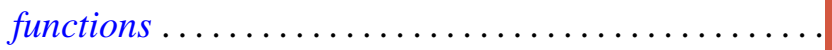

771

785

Hans H. Storrer, Rational extensions of modules

795

topological lattices ...........................

Robert Evert Stong, On the cobordism of pairs .................. 803
Albert Leon Whiteman, An infinite family of skew Hadamard matrices . . . 817

Lynn Roy Williams, Generalized Hausdorff-Young inequalities and mixed

norm spaces 